\title{
PAX3 silencing suppresses gastric cancer proliferation and angiogenesis via MET/PI3K signaling
}

\author{
W. L. LV ${ }^{1}$, Y. Y. HU², Z. N. LI ${ }^{3}$, W. ZHANG ${ }^{4}$, Q. PAN ${ }^{5, *}$ \\ ${ }^{1}$ Department of Anatomy and Physiology, Lianyungang TCM Branch of Jiangsu Union Technical Institute, Lianyungang, Jiangsu, China; \\ ${ }^{2}$ Department of Human Anatomy, Kangda College of Nanjing Medical University, Lianyungang, Jiangsu, China; ${ }^{3}$ Department of Neurology, \\ Second Affiliated Hospital of Xuzhou Medical University, Xuzhou, Jiangsu, China; ${ }^{4}$ Department of Neurology, Affiliated Hospital of Xuzhou \\ Medical University, Xuzhou, Jiangsu, China; ${ }^{5}$ Department of General Surgery, Ru Shan People's Hospital, Weihai, 264500, Shandong, China
}

*Correspondence: slxldpb8839413@126.com

Received April 29, 2019 / Accepted July 23, 2019

\begin{abstract}
PAX3 is the key factor in cell signal transduction pathway and may be involved in the regulation of cancer cell proliferation, differentiation and migration. The aim of the study was to investigate the effects and mechanism of PAX3 silencing on the gastric cancer. Specific PAX3 silencing was performed both in vitro and in vivo using small-interfering RNAs (siRNAs). The proliferation, apoptosis and angiogenesis of gastric cancer cells were assessed using MTT assay, flow cytometry and in vitro tube formation assay. Mice with gastric xenografts, which expressed either si-PAX3 or non-coding siRNA (si-NC), were developed and the effects of PAX3 silencing on tumor progression were evaluated. PCNA is a proliferating cell nuclear antigen and can be used as an index for evaluating cell proliferation status. Immunocytochemistry assay was used to quantify PAX3 and PCNA expression. After 4 weeks of tumor inoculation, tumor tissues were weighed. Tumor tissue morphology and apoptosis were evaluated using HE staining and TUNEL assay. In order to investigate the effect of silencing PAX3 on cell apoptosis, angiogenesis and MET/PI3K pathway, quantitative real-time PCR (qRT-PCR) or western blot were used to detect the expression levels of caspase-3, VEGF, MET, p-MET, PI3K and p-PI3K. After PAX3 silencing, PAX3 expression was significantly decreased in two gastric cancer cell lines, MKN-28 and SGC-7901 ( $\mathrm{p}<0.05$ vs Control). PAX3 silencing reduced cell proliferation, induced cell apoptosis and inhibited tube formation. PAX3 and PCNA expression were also significantly decreased. In mice, silencing PAX3 significantly inhibited tumor growth and decreased microvessel density in tumor. PAX3 silencing also decreased cell density in tumors, which concurred with increased apoptosis and PAX3 expression. PAX3 silencing upregulated the expression of caspase-3, downregulated the expression of VEGF, phosphorylation of PI3K and MET. Our data showed that these anti-tumor effects of PAX3 silencing might be attributed to its role in inducing cell apoptosis and inhibiting angiogenesis.
\end{abstract}

Key words: PAX3, angiogenesis, gastric cancer

Gastric cancer is ranked fourth cause of cancer related mortality worldwide and the most common cause of cancerrelated death in the world [1]. Metastasis has been identified as the primary reason for the malignant progression and high mortality of gastric cancer patients. However, the molecular mechanisms underlying gastric metastasis have not yet been fully explained.

The paired box $(P A X)$ is a gene family comprised of nine members both in man (PAX1 to PAX 9) and mouse (pax1 to pax 9 ). They are further classified into three sub-categories based on structural similarity such as homeodomain, paired domain and octapeptide [2], PAX gene family has recently been recognized to play a crucial role in cell proliferation, differentiation, migration and during embryonic development [3]. A potential link has also been demonstrated to exist between aberrant expression of $P A X 3$ in adult tissues and a wide variety of cancers by promoting or inhibiting tumorigenesis $[2,4]$. A good correlation between the PAX3 gene and oncogenesis has been established [5]. PAX has been shown to be upregulated with an increased expression in neuroblastomas, glioblastomas, rhabdomyosarcomas, melanomas, Ewing's sarcomas and gastric cancers [6-8].

It was demonstrated that the paired box-3 (PAX3) and met proto-oncogene (MET) were overexpressed in gastric cancer, and these seemed to be regulated by miRNA [9]. On the other hand, Zhang et al. showed that miR-206 significantly suppressed gastric cancer cell invasion and metastasis both in vitro and in vivo by downregulating PAX3. In vivo metastasis assay showed that overexpression of PAX3 promoted invasiveness and pulmonary metastasis [10]. Therefore, 
PAX3 is an oncogene of high interest in the study of gastric cancer.

To unravel the mechanism of PAX3 in cancer regulation, Misuraca et al. showed that PAX3 acted in a p53 dependent pathway to inhibit cell apoptosis of brainstem progenitor cells [11]. Chen et al. showed that PAX3 was upregulated in tissue specimens of glioblastoma (GBM) with high-grade rather than low-grade or normal. The PAX3 was highly expressed with the increase in tumor WHO grade [12]. They further demonstrated that hypoxia inducible factor-1a (HIF-1a) mediated PAX3/p53 axis, giving insight to the mechanism that controls self-renewal, migration, and tumorigenesis of brain glioma stem cells (BGSCs) in a p53-dependent manner [13]. Liu et al. demonstrated that PAX3 played a role as an oncosuppressor in the development of thyroid cancer. Their study showed that PAX3 was downregulated by promoting methylation in both primary thyroid tissue and thyroid cell lines [14]. The oncosuppressing activity was shown to be through the inhibition of cell growth and invasiveness in thyroid cancer cells through repressing the activities of PI3K/ Akt and MAPK/Erk pathways and promotion of FOXO3a activity $[14,15]$. PAX3 has also been shown to be upregulated in Ewing's sarcoma [16], neuroblastomas [17], rhabdomyosarcomas [18] and melanomas.

The mechanisms in which PAX is involved in development of cancers seem varied for the different cancer types. In rhabdomyosarcoma and other soft tissue sarcomas, pax transcription factors appear to increase the proliferation rate while decrease the amount of apoptosis in tumor cells. This can be a result of regulation of different target genes like p53, bcl-2, and receptor tyrosine kinases [19].

The current study aimed at further understanding the effects and mechanism of PAX3 silencing on proliferation, apoptosis and angiogenesis of gastric cancer. Specific PAX3 silencing was performed both in vitro and in vivo using small-interfering RNAs (siRNAs).

\section{Materials and methods}

Animals. Animal experiments followed the NIH guidelines (NIH Pub. No. 85-23, revised 1996) and were reviewed and approved by the Animal Protection and Use Committee of Lianyungang TCM Branch of Jiangsu Union Technical Institute. Balb/c male nude mice (7-8 weeks old, $20 \pm 2 \mathrm{~g}$ ) were acquired from Jinan Peng Yue experimental animal breeding Co. (license number SCXK (Lu) 2014-0007) and housed in an SPF-grade room maintained at the temperature at $22-24^{\circ} \mathrm{C}$, with $50-60 \%$ relative humidity.

Cell culture. Human gastric cancer cell lines, MKN-28 (BNCC102156) and SGC-7901 (BNCC100674) were cultured in RPMI 1640 medium (Gibco) supplemented with 10\% fetal bovine serum (Sigma-Aldrich, St. Louis, MO, USA). Human umbilical vein endothelial cells (HUVECs) were acquired from American Type Culture Collection (PCS-100013, Rockville, MD, USA) and cultured in DMEM medium
(Gibco, Invitrogen, USA) containing $4.5 \mathrm{~g} / \mathrm{l} \mathrm{D}$-glucose, L-glutamine and $110 \mathrm{mg} / \mathrm{l}$ sodium pyruvate. All cells were cultured in a humid incubator maintained at $37^{\circ} \mathrm{C}, 5 \% \mathrm{CO}_{2}$ (Thermo Fisher Scientific, Waltham, USA). Cells at the logarithmic growth phase were used for studies.

Cell transfection. Cells $\left(2 \times 10^{5}\right.$ cells $)$ were seeded in 6-well plates. When the cells reached $80 \%$ confluence, Lipofectamine $2000^{\circ}$ reagent (Invitrogen; Thermo Fisher Scientific, Inc.) was used to transfect the cells with PAX3-siRNA (GAAACACCGTGCCGTCAGT) or control-siRNA (TTCTCCGAACGTGTCACGT) (cat. no. 215907; Invitrogen; Thermo Fisher Scientific, Inc.) in accordance with the manufacturer's recommendations. The duration of transfection was $24 \mathrm{~h}$. qRT-PCR was used to evaluate transfection efficiency. Primers for PAX3 were designed as follows: F: 5'-TGCATCCAAATTCGGAGTAG-3'; R: 5'-TGAGTCCACATCGGATGTGTCG-3'. The following groups were evaluated: (1) normal control group (Control): normal culture of gastric cancer cells; (2) PAX3 silencing group (si-PAX3): cells transfected with PAX3 siRNA; (3) siRNA transfection negative control group (si-NC): cells transfected with non-coding siRNA.

MTT assay. Cells were trypsinized and diluted to the concentration of $1 \times 10^{5} / \mathrm{ml}$ and seeded into a 96-well plate. After incubation for $24 \mathrm{~h}, 48 \mathrm{~h}$, and $72 \mathrm{~h}, 20 \mu \mathrm{l}$ of MTT $(5 \mathrm{mg} / \mathrm{ml})$ reagent was added. After $4 \mathrm{~h}, 200 \mu \mathrm{l}$ DMSO was added (Sigma-Aldrich, St. Louis, MO, USA). The absorbance (OD value) of each well was measured at the wavelength of $490 \mathrm{~nm}$ in a microplate reader (Bio-Rad Laboratories).

Flow cytometry. Cell suspensions $\left(1 \times 10^{6} / \mathrm{ml}\right)$ were incubated with $5 \mu \mathrm{l}$ FITC-conjugated Annexin-V, gently mixed for $3 \mathrm{~min}$. Ten $\mu \mathrm{l}$ of propidium iodide (PI, $20 \mu \mathrm{g} / \mathrm{ml}$ ) was then added and incubated for $10 \mathrm{~min}$ at room temperature in the dark. After adding another $400 \mu$ of $1 \times$ binding buffer, cells were evaluated in a flow cytometer (Galios; Beckman Coulter, Inc, Brea, California, USA) equipped with Cell Quest software (BD Biosciences, San Diego, CA, USA).

In vitro tube formation assay. 96-well plates were coated with $50 \mu \mathrm{l}$ Matrigel (BD Bioscience, USA) by incubation for $5 \mathrm{~h}$ at $37^{\circ} \mathrm{C}$. The HUVEC cells seeded into coated plates at the density of $4 \times 10^{4}$ cells/well. After a $6 \mathrm{~h}$ incubation, the tube formation was evaluated under an Olympus inverted microscope (CKX40, Olympus, New York, NY).

Immunohistochemistry in vitro. Cells were fixed with paraformaldehyde for $30 \mathrm{~min}$, washed with PBS for 2-3 times, and treated with $0.5 \%$ Triton X-100 for $20 \mathrm{~min}$. After washing, $3 \% \mathrm{H}_{2} \mathrm{O}_{2}$ was added and incubated with the cells for 15-20 min. Bovine serum albumin (BSA) was then added and incubated for $30 \mathrm{~min}$ at $37^{\circ} \mathrm{C}$ to block non-specific binding. Primary antibodies, including rabbit anti-human anti-PAX3 antibody (1: 100 dilution, ab180754, Abcam, UK) and anti-PCNA Antibody (1: 100 dilution, ab15497, Abcam, UK) were then used to incubate with the cells at $37^{\circ} \mathrm{C}$ for $30 \mathrm{~min}$. After washing with PBS for 2-3 times, horseradish peroxidase (HRP)-conjugated goat anti-rabbit IgG antibody (Proteintech, USA) was added and incubated with the cells at 
$37^{\circ} \mathrm{C}$ for $30 \mathrm{~min}$, followed by adding DAB. Stained cells were examined under an optical microscope (Olympus, Japan), using 3-5 randomly selected fields of view for quantification.

Construction of mouse xenograft models. $200 \mu \mathrm{l}$ of cell suspension at the concentration of $1 \times 10^{7} \mathrm{cell} / \mathrm{s} / \mathrm{ml}$ were subcutaneously injected to BALB/c male nude mice. The following experimental groups were used: (1) normal group (Control): normal feeding of mice, no treatment; (2) the model group (Model): normal gastric cancer cell suspension was inoculated; (3) silencing PAX3 expression group: inoculation of cell suspension transfected with si-PAX3; (4) negative control group: inoculation of cell suspension transfected with si-NC. Mice were observed daily for survival. Tumor growth was observed weekly by caliper measurements and tumor volume size; tumor volume was calculated as follows: $\mathrm{V}=\mathrm{a}^{\star} \mathrm{b}^{2} / 2$ (a: length; b: width). At four weeks after tumor inoculation, intraperitoneal injection of sodium pentobarbital $(50 \mathrm{mg} / \mathrm{kg})$ was performed to anesthetize the mice, followed by cervical dislocation. The tumors were weighed. A portion of the tumor tissue was fixed in 4\% paraformaldehyde solution and used for subsequent HE staining and TUNEL staining. Part of the tumor tissue was immersed into liquid nitrogen and then transferred to the $-80^{\circ} \mathrm{C}$, and later used for qRT-PCR and western blot detection.

HE staining. Fixed tissues were paraffin-embedded and sliced at the thickness of $5 \mu \mathrm{m}$. The tissue slides were dewaxed and rehydrated using gradient ethanol. The slides were then immersed in hematoxylin (Solarbio, Beijing, China) for 5 min, rinsed with tap water, treated with $\mathrm{HCl}$ for $30 \mathrm{~s}$, washed with tap water for $15 \mathrm{~min}$, and placed in Yihong Dyeing Liquid (Solarbio, Beijing, China) for 2 min. Slides underwent conventional dehydration procedures and mounted with coverslips. Staining was observed under a 400× optical microscope (Olympus type BX51, Olympus, Japan).

TUNEL assay in vivo. Tissue slides were stained with the apoptosis detection kit (lot: ZK-8005, Beijing Zhongshan Golden Bridge Biotechnology Co., Ltd., China). To quantify apoptosis, stained tissues were examined using a microscope under $400 \times$ based on 5 randomly used field. Normal nuclei were stained blue and positive apoptotic cells were stained brown.

Immunohistochemical staining of PAX3 to evaluate tumor microvessel density (MVD). Immunohistochemical detection of PAX3 was performed using rabbit anti-rat anti-PAX3 antibody (1:100 dilution, ab180754, Abcam, UK). $\mathrm{SABC}$ immunohistochemistry kit was used with $\mathrm{DAB}$ as the substrate. Rabbit anti-mouse anti-CD31 antibody (1: 50 dilution, Ab28364, Abcam, UK) was used to stain microvessel. MVD was determined using the Weidner counting method. The high vascular density (hot-spot) was determined under a 100× magnification, and the MVD of 5 fields was counted under a $400 \times$ field of view. The mean value was taken as the MVD value of the tumor.

Quantitative RT-PCR. After homogenizing the tissue, total RNAs were extracted using the Trizol agent (15596018,
Invitrogen, Carlsbad, CA, USA), followed by centrifugation at $10000 \times \mathrm{g}$ for $10 \mathrm{~min}$ at $4{ }^{\circ} \mathrm{C}$. The Reverse Transcription kit (Applied Biosystems, Waltham, MA, USA) was used for cDNA synthesis. qRT-PCR was performed using a Mastercycler ${ }^{\oplus}$ nexus X2 (Eppendorf, Hamburg, Germany). Data were processed by the $2^{-\Delta \Delta C t}$ method using GAPDH mRNA as an internal control. The primers (Shanghai Shenggong Bioengineering Technology Service Co., Ltd.) used were as follows: Caspase-3: Forward: 5'-GTGCTATTGTGAGGCGGTT-3'; Reverse: 5'-CGAGATGTCATTCCAGTGCT-3'; VEGF: Forward: 5'-GTTACCTCAGCAAGACGTTGT-3', Reverse: 5'-AGGAAGTGTGATTGGCAAAACT-3'; GAPDH: Forward: 5'-AGAAGGCTGGGGCTCATTTG-3', Reverse: 5'AGGGGCCATCCACAGTCTTC-3'.

Western blot. The cells were lysed in RIPA buffer and the BCA method (Pierce ${ }^{\mathrm{m} t}$ BCA Protein Assay Kit, Thermo Fisher Scientific, Waltham, USA) was used for measuring the protein concentration. Protein lysates of $40 \mu \mathrm{g}$ were resolved using SDS-PAGE electrophoresis (Mini-Protean-3 Type, Bio-Rad, Hercules, CA, USA), followed by transferring to PVDF membrane (Merck, Darmstadt, Germany). Membranes were blocked with $5 \%$ skim milk powder in TBST for $1 \mathrm{~h}$. Rabbit anti-mouse anti-Caspase-3 antibody (1: 500, ab13847, Abcam, UK ), anti-VEGF antibody (1:1000, ab53465, Abcam, UK), anti-MET antibody (1:1000, ab74217, Abcam, UK ), anti-p-MET antibody (1:1000, ab7 3992, Abcam,UK ), anti-p-PI3K antibody (1:1000, ab182651, Abcam, UK), anti-PI3K antibody (1:2000, ab227204, Abcam, UK ) or $\beta$-actin (1:1000, ab8227, Abcam, UK) were added and incubated for $1 \mathrm{~h}$ at RT. Goat anti-rabbit $\operatorname{IgG}$ (1:2000, ab6721, Abcam, UK) was then added. ECL chemiluminescence method was used for detecting protein bands and the protein expression level was normalized by $\beta$-actin. Quantification was performed using the ImageJ (NIH) software.

Statistical method. Application SPSS19.0 software was used for statistical analysis. Data were expressed as mean \pm standard deviation (mean $\pm \mathrm{SD}$ ). Differences between multiple groups of data were analyzed using one-way ANOVA Analysis (ANOVA). The differences were considered statistically significant at $\mathrm{p}<0.05$.

\section{Results}

PAX3 silencing inhibited cell proliferation and angiogenesis in vitro. The efficiency of PAX3 siRNA transfection in MKN-28 and SGC-7901 cells was evaluated by qRT-PCR and western blot (Figure 1A, 1B). Compared with control group, the PAX3 levels were significantly decreased in si-PAX3 group $(\mathrm{p}<0.05)$. For further experiments, we chose MKN-28 cells because of high transfection efficiency observed. At $24 \mathrm{~h}, 48 \mathrm{~h}$ and $72 \mathrm{~h}$ after transfection, significant reduction in cell proliferation was observed in MKN-28 cells transfected with si-PAX3 compared to non-transfected cells $(\mathrm{p}<0.05$, Figure $1 \mathrm{C})$. Apoptosis of MKN-28 cells following si-PAX3 or si-NC transfection was detected by flow cytom- 
A
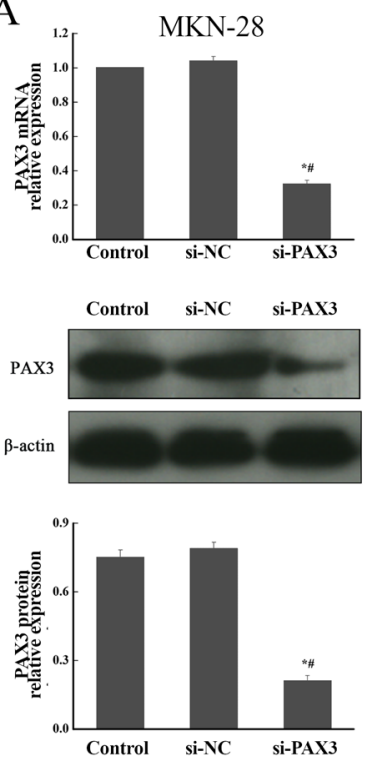
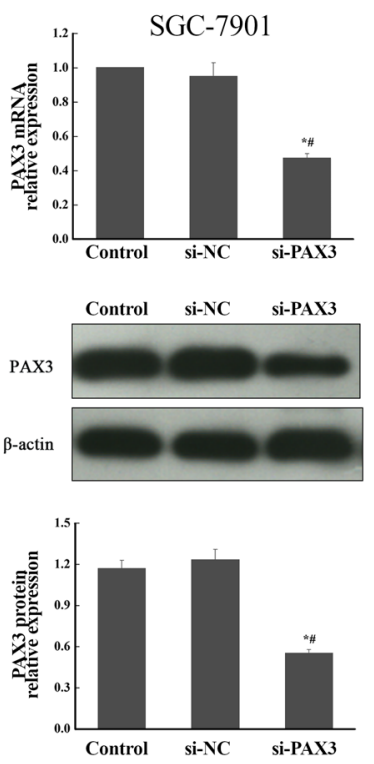

B

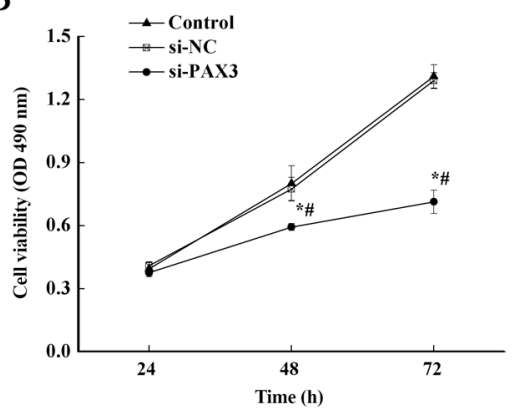

$\mathrm{C}$
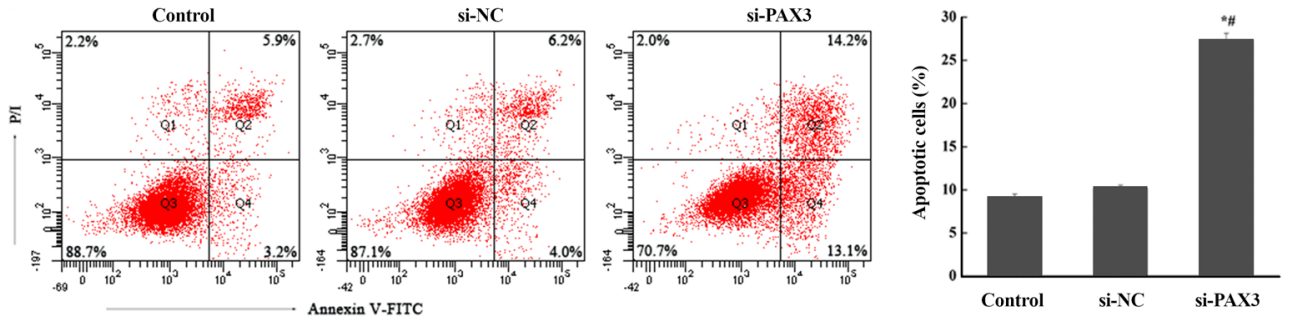

$\mathrm{D}$
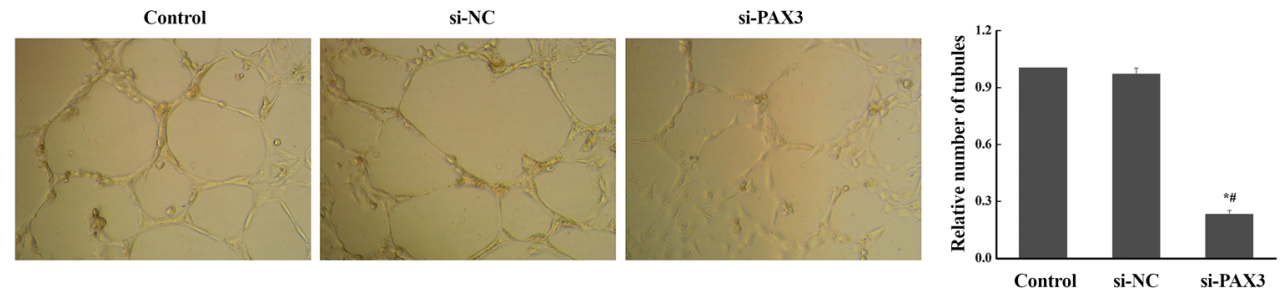

Figure 1. PAX3 silencing inhibited cell proliferation and angiogenesis. A) qRT-PCR and western blot detection of PAX3 after transfecting si-NC or siPAX3. B) PAX3 silencing reduced proliferation of MKN-28 cells. C) PAX3 silencing induced gastric cell apoptosis. D) The microvessel density (MVD) was measured by tube formation in MKN-28 cells transfected with si-NC or si-PAX3 (400 $\times)$. ${ }^{*} \mathrm{p}<0.05$ compared with the Control group; \#p $<0.05$ compared with the si-NC group.

etry after Annexin-FITC/PI staining (Figure 1D). It was shown that compared with non-transfected cells, si-PAX3 transfection elevated cell apoptosis significantly $(p<0.05)$. We next examined the effects of PAX3 silencing on angiogenesis of MKN-28 cells. As shown in Figure 1E, silencing of PAX3 induced pronounced decrease of tube formation. This data indicated that PAX3 silencing was effective in attenuating angiogenesis in vitro.

PAX3 silencing reduced PAX3 and PCNA protein expression in vitro. Immunohistochemistry was used to evaluate PAX3 and PCNA expression in MKN-28 cells. As shown in Figure 2, the densities of positively-stained PAX3 and PCNA in si-PAX3 group were significantly decreased
( $\mathrm{p}<0.05$, red arrows), suggesting that silencing PAX3 expression could downregulate PAX3 and PCNA protein expression in MKN-28 cells.

PAX3 silencing impeded gastric tumor growth in vivo. MKN-28 cells, transfected with si-NC or si-PAX3, were used to initiate tumor xenografts in mice. Non-transfected MKN-28 cells were also used to initiate tumors, which were used as controls (model group). Starting from day 5 after inoculation, model and si-NC groups showed prominently reduced tumor growth rate $(\mathrm{p}<0.05$, Figure $3 \mathrm{~A})$ compared to the rapid tumor growth tumors with si-PAX3 transfection. Mice were sacrificed after 4 weeks and tumors were harvested. Consistent with the reduction in tumor growth, 

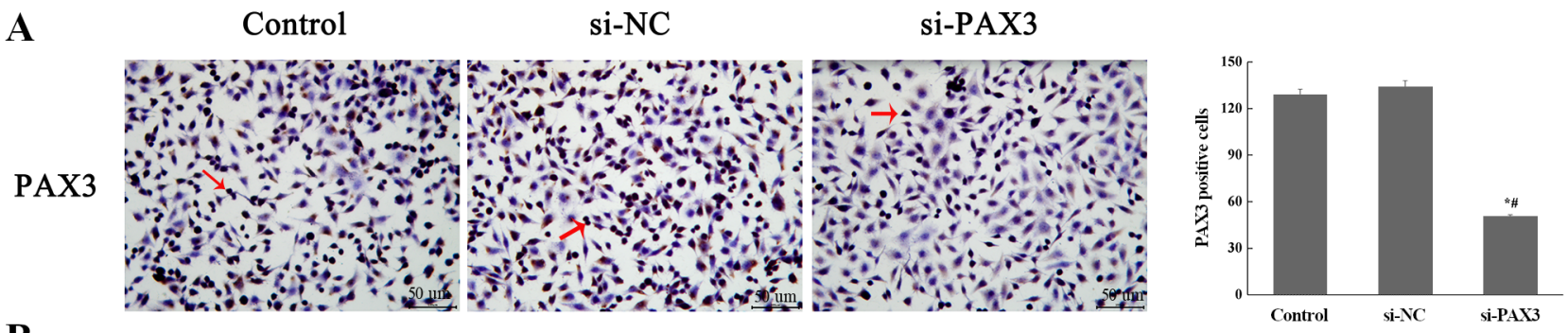

B
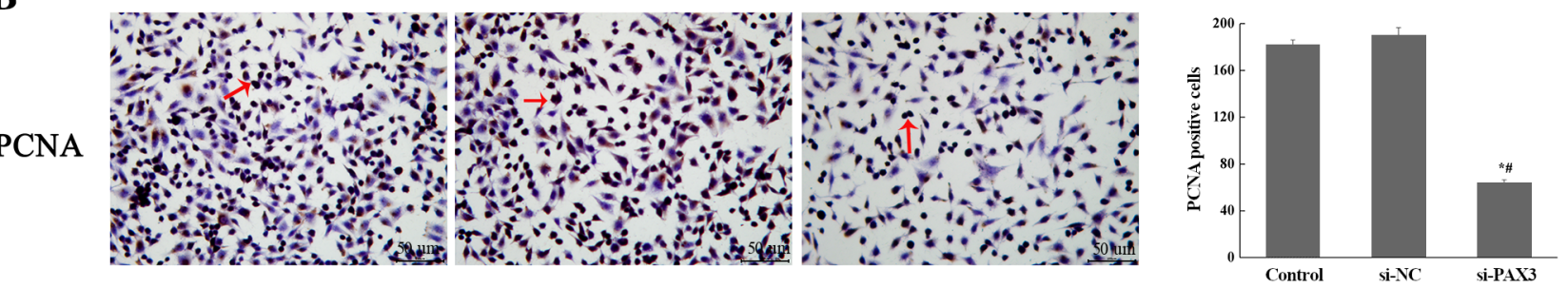

Figure 2. PAX3 silencing reduced PAX3 and PCNA protein expression in vitro. Representative immunohistochemical staining photograph (400 $\times)$ and quantification of PAX3 (A) and PCNA (B). ${ }^{*} \mathrm{p}<0.05$ compared with the Control group; \# $\mathrm{p}<0.05$ compared with the si-NC group.

A

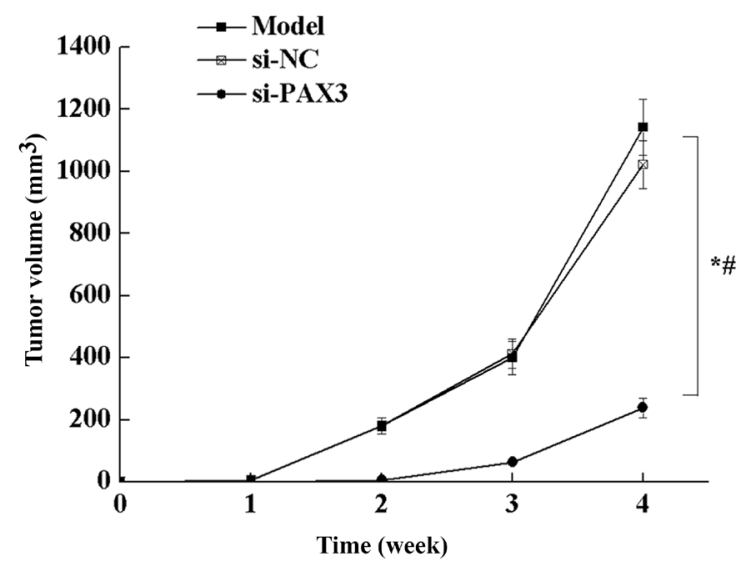

B

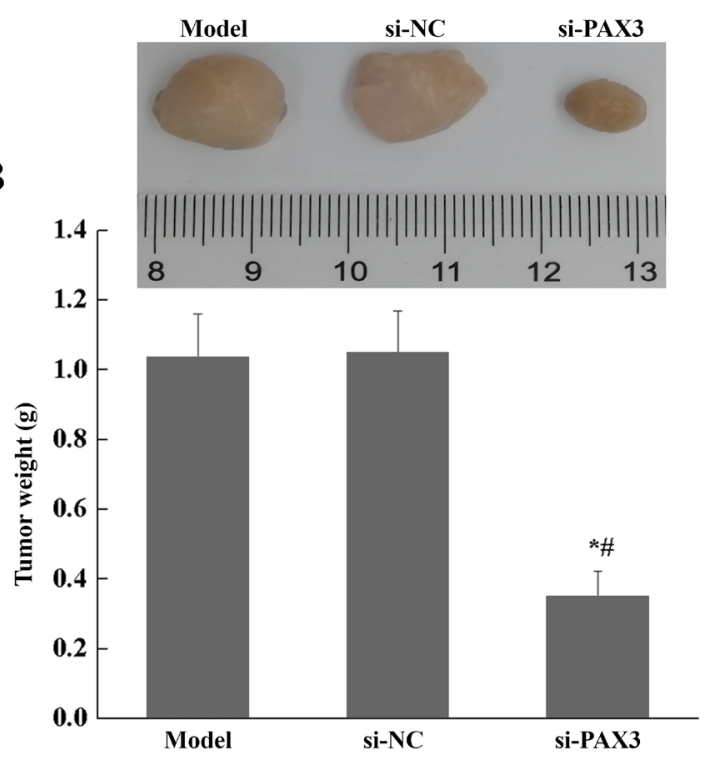

Figure 3. PAX3 silencing impeded gastric tumor growth in vivo. A) Volume change of transplanted tumor in nude mice. B) Weight of transplanted tumor in nude mice. ${ }^{\star} \mathrm{p}<0.05$, compared with Model group; $\# \mathrm{p}<0.05$, compared with si-NC group.

si-PAX3 group also showed a significantly lower tumor weight when compared to model group and si-NC group $(\mathrm{p}<0.05$, Figure 3B).

PAX3 silencing reduced cell density and increased tumor cell apoptosis in transplanted tumor. To explore the cause of reduced tumor growth after si-PAX3 transfection, $\mathrm{HE}$ staining was used to investigate the morphological changes of tumor (Figure 4A). Tumor cells density in the si-PAX3 group was significantly reduced, which correlated well with the attenuated tumor growth. TUNEL staining unveiled that number of apoptotic cells and number of apoptotic cells was dramatically increased in si-PAX3 group (Figure $4 \mathrm{~B}$, red arrows, $\mathrm{p}<0.05$ ). This evidence suggested that silencing PAX3 expression could induce tumor cell apoptosis.

PAX3 silencing reduced PAX3 expression and tumor microvessel density in vivo. As shown in Figure 5A, PAX3 was mainly expressed in the nucleus and weakly expressed in the cytoplasm. Compared to model group, si-PAX3 group demonstrated significantly reduced expression of PAX3 (Figure 5A) along with decreased MVD (Figure 5B) $(\mathrm{p}<0.05)$, suggesting that silencing PAX3 expression could reduce tumor microvascular density and inhibit angiogenesis. 
A

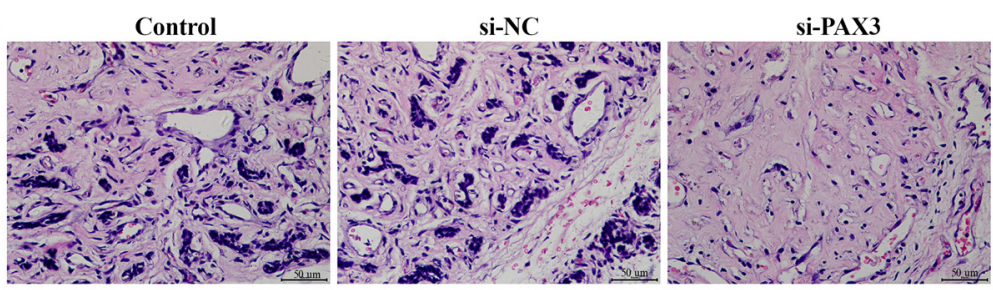

B
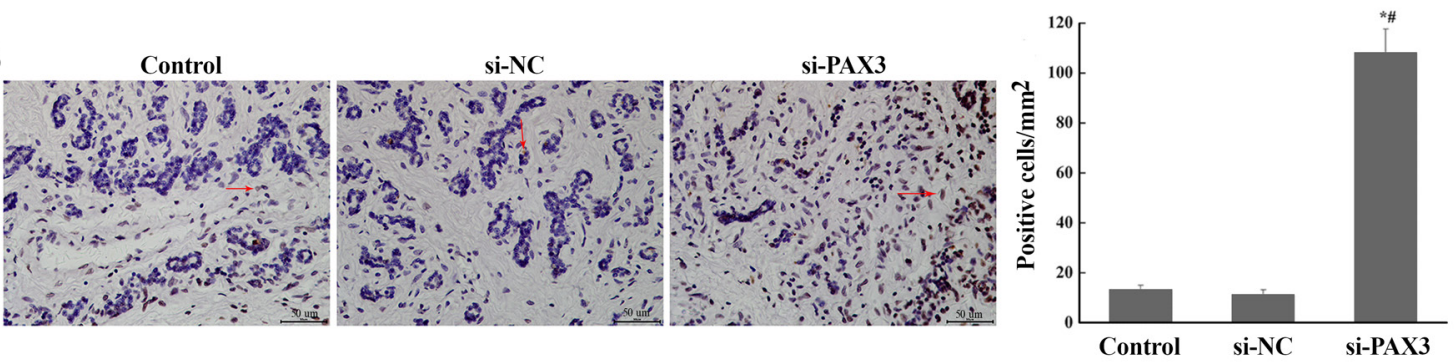

Figure 4. PAX3 silencing reduced cell density and increased tumor cell apoptosis in transplanted tumor. A) HE staining of nude mice xenografts $(400 \times)$. B) TUNEL assay for tumor cell apoptosis $(400 \times) .{ }^{\star} \mathrm{p}<0.05$, compared with control group; $\# \mathrm{p}<0.05$, compared with si-NC group.
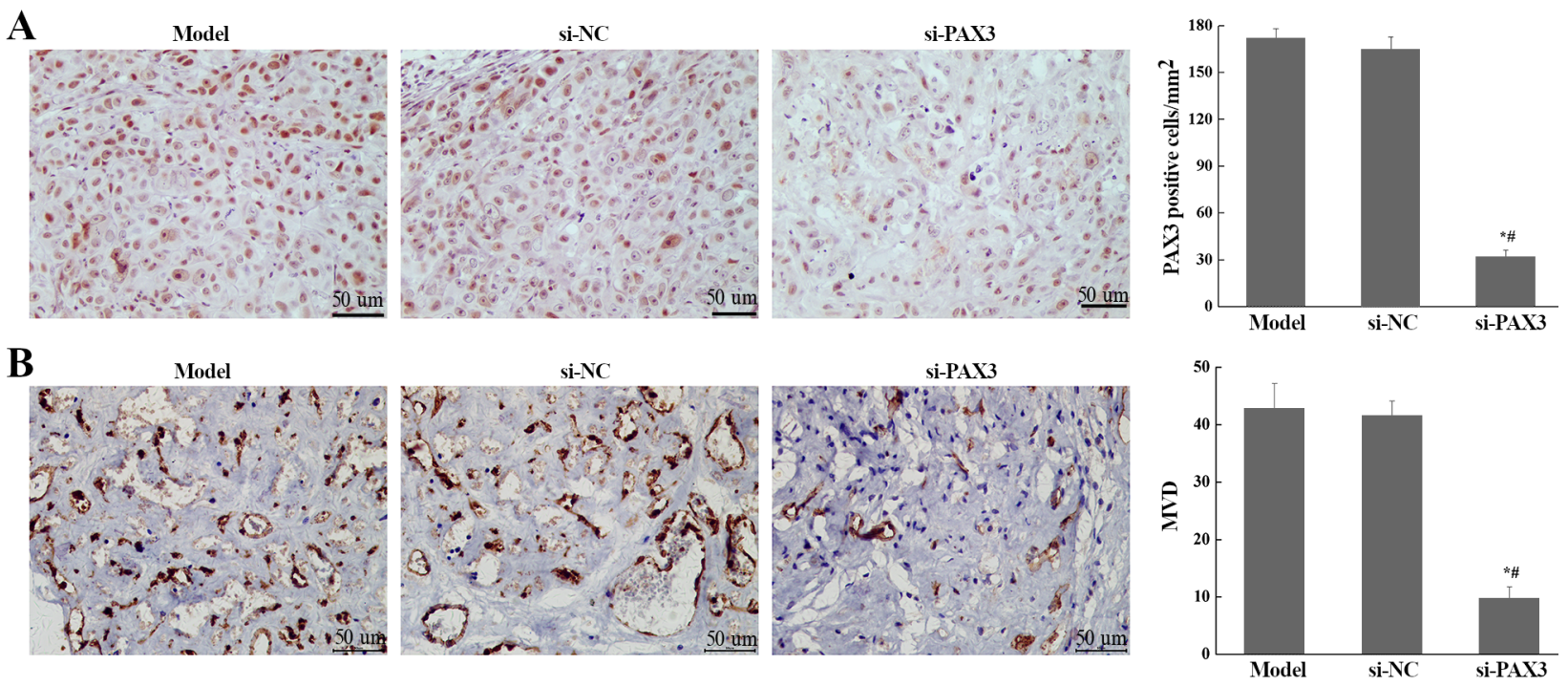

Figure 5. Detection of PAX3 and MVD using immunohistochemical staining in tumor tissues. A) PAX3 immunohistochemistry and quantitative analysis of positively stained cells $(400 \times)$. B) CD31 immunohistochemistry and MVD values in tumor blood vessels $(400 \times)$. ${ }^{*} p<0.05$, compared with control group; \#p<0.05, compared with si-NC group.

Silencing of PAX3 increased caspase-3, decreased VEGF and attenuated MET/PI3K signaling in vivo. As shown in Figure 6A, qRT-PCR revealed that no significant differences could be observed in caspase-3 and VEGF mRNA levels between si-NC and model group ( $>>0.05$ ), while si-PAX3 group demonstrated significantly increased caspase- 3 and decreased VEGF mRNA when compared with si-NC group $(p<0.05)$. Western blot analysis confirmed the changes of caspase- 3 and VEGF levels induced by PAX3 silencing on protein level (Figure 6B). Further, MET/PI3K signaling was attenuated by PAX3 silencing, evidenced by reduction in the expression of $\mathrm{p}-\mathrm{MET}$ and $\mathrm{p}$-PI3K levels, compared to the model group $(\mathrm{p}<0.05)$. Therefore, silencing of PAX3 was effective in attenuating MET/PI3K signaling and reducing angiogenesis, which could be a mechanism of PAX3 silencing in inhibiting gastric cancer.

\section{Discussion}

Our results showed that after PAX3 expression was significantly decreased in two gastric cancer cell lines, MKN-28 and SGC-7901 as a result of PAX3 silencing. Furthermore, silence 

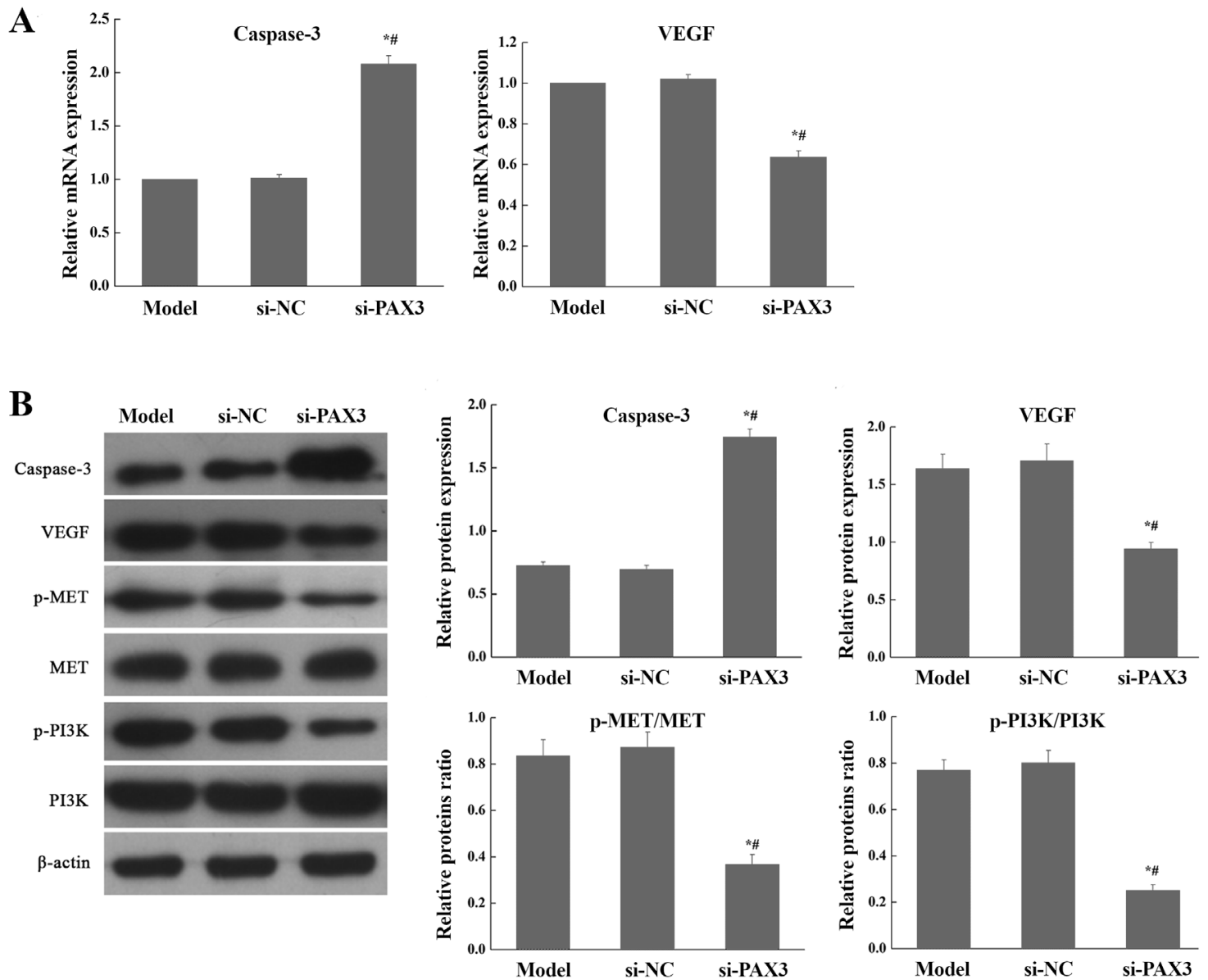

Figure 6. Silencing of PAX3 increased caspase-3, decreased VEGF and attenuated MET/PI3K signaling. A) qRT-PCR detection of caspase-3 and VEGF in tumor tissues. B) Levels of caspase-3, VEGF and MET/PI3K signaling by western Blot. ${ }^{\star} \mathbf{p}<0.05$, compared with control group; \#p $<0.05$, compared with si-NC group.

of PAX3 could suppress gastric cancer growth through inhibiting VEGF and phosphorylation of MEK/PI3K pathway.

In previous studies, suppression of PAX3 has been reported to use different gene targets. Li et al. reported the suppression of PAX3 using PTEN tumor suppressor gene, which affects the PTEN/AKT survival pathway [20]. Gryder et al. showed that PAX3-FOXO1 recruited the BET bromodomain protein BRD4 to play a role in supper enhancers and had a significant sensitivity to BRD inhibition [21]. Our results demonstrated another pathway of PAX3 suppression by siRNA. Si-PAX3 transfection attenuated cell proliferation of cancer cell lines, thus supporting previous observations of other suppression mechanisms. It has previously been shown that siRNA molecules effectively suppressed the proliferation of cancer cells to different extents, and also knocked down their cognate targets such as bcl-2, mdm-2, pkc-alpha, cdk-2tgf-beta1, H-ras, vegf, and GFP mRNAs [22].

With regards to tumor development, we found that PAX3 silencing impeded gastric tumor growth in vivo, resulted from reducing cell density in transplanted tumor cells and reduced tumor microvessel density. These data collectively pointed to a potential role of PAX3 in the development of gastric cancers. Previous in vivo metastasis studies demonstrated that overexpression of PAX3 significantly promoted invasiveness and pulmonary metastasis of gastric cancer cells [23]. Our results concurred with this observed phenomenon. We also examined the role of PAX3 in apoptosis and found that PAX3 silencing increased tumor cell apoptosis as evidenced by TUNEL staining, which revealed that apoptotic cells in the si-PAX3 group were dramatically increased as compared to its control, thus supporting the argument that PAX3 suppression did induce tumor apoptosis. We further demonstrated an increase in caspase- 3 and a decrease in VEGF levels induced by PAX3 silencing on protein level. Further, MET/PI3K signaling was attenuated by PAX3 silencing, as evidenced by reduction in the expression of p-MET and p-PI3K levels. In rhabdomyosarcoma and other tissue sarcomas, PAX has been shown to reduce the amount 
of apoptosis in tumor cells, with the proposed mechanism being through the regulation of different target genes such as p53, bcl-2, and receptor tyrosine kinases $[18,19]$. Our results showed that silencing of PAX3 was effective in attenuating MET/PI3K signaling and reducing angiogenesis and this could be a mechanism of PAX3 silencing in inhibiting gastric cancer.

In conclusion, the results from the current study showed that silencing of PAX3 expression could inhibit the proliferation, angiogenesis of gastric cancer cells and induce apoptosis. The mechanism might be through upregulation of the expression of the pro-apoptotic protein caspase-3, downregulation of VEGF, and inhibition of its downstream MET/ PI3K signaling.

\section{References}

[1] FERLAY J, SHIN HR, BRAY F, FORMAN D, MATHERS C et al. Estimates of worldwide burden of cancer in 2008: GLOBOCAN 2008. Int J Cancer 2010; 127: 2893-2917. https:// doi.org/10.1002/ijc. 25516

[2] ROBSON EJ, HE SJ, ECCLES MR. A PANorama of PAX genes in cancer and development. Nat Rev Cancer 2006; 6: 52-62. https://doi.org/10.1038/nrc1778

[3] SCHAFER BW. Emerging roles for PAX transcription factors in cancer biology. Gen Physiol Biophys 1998; 17: 211-224.

[4] ARASU A, MURUGAN S, ESSA MM, VELUSAMY T, GUILLEMIN GJ. PAX3: a molecule with oncogenic or tumor suppressor function is involved in cancer. BioMed Res Int 2018; 1095459. https://doi.org/10.1155/2018/1095459

[5] WANG Q, FANG WH, KRUPINSKI J, KUMAR S, SLEVIN $\mathrm{M}$ et al. Pax genes in embryogenesis and oncogenesis. J Cell Mol Med 2008; 12: 2281-2294. https://doi.org/10.1111/ j.1582-4934.2008.00427.x

[6] XIA L, HUANG Q, NIE D, SHI J, GONG M et al. PAX3 is overexpressed in human glioblastomas and critically regulates the tumorigenicity of glioma cells. Brain Res 2013; 1521: 68-78. https://doi.org/10.1016/j.brainres.2013.05.021

[7] FANG WH, WANG Q, LI HM, AHMED M, KUMAR P et al. PAX3 in neuroblastoma: oncogenic potential, chemosensitivity and signalling pathways. J Cell Mol Med 2014; 18: 38-48. https://doi.org/10.1111/jcmm.12155

[8] BARR FG, GALILI N, HOLICK J, BIEGEL JA, ROVERA G et al. Rearrangement of the PAX3 paired box gene in the paediatric solid tumour alveolar rhabdomyosarcoma. Nat Genet 1993; 3: 113-117. https://doi.org/10.1038/ng0293-113

[9] WU Y, WAN X, JI F, SONG Z, FANG X. Serum miR-658 induces metastasis of gastric cancer by activating PAX3-MET pathway: A population-based study. Cancer Biomark 2018; 22: 111-118. https://doi.org/10.3233/CBM-171045

[10] ZHANG L, LIU X, JIN H, GUO X, XIA L et al. miR-206 inhibits gastric cancer proliferation in part by repressing cyclinD2. Cancer Lett 2013; 332: 94-101. https://doi. org/10.1016/j.canlet.2013.01.023
[11] MISURACA KL, BARTON KL, CHUNG A, DIAZ AK, CONWAY SJ et al. Pax3 expression enhances PDGF-B-induced brainstem gliomagenesis and characterizes a subset of brainstem glioma. Acta Neuropathol Commun 2014; 2: 134. https://doi.org/10.1186/s40478-014-0134-6

[12] CHEN J, XIA L, WU X, XU L, NIE D et al. Clinical significance and prognostic value of PAX3 expression in human glioma. J Mol Neurosci 2012; 47: 52-58. https://doi. org/10.1007/s12031-011-9677-1

[13] ZHU H, WANG H, HUANG Q, LIU Q, GUO Y et al. Transcriptional Repression of p53 by PAX3 Contributes to Gliomagenesis and Differentiation of Glioma Stem Cells. Front Mol Neurosci 2018; 11: 187. https://doi.org/10.3389/ fnmol.2018.00187

[14] LIU W, SUI F, LIU J, WANG M, TIAN S et al. PAX3 is a novel tumor suppressor by regulating the activities of major signaling pathways and transcription factor FOXO3a in thyroid cancer. Oncotarget 2016; 7: 54744-54757. https://doi. org/10.18632/oncotarget.10753

[15] LIU Q, YANG G, QIAN Y. Loss of MicroRNA-489-3p promotes osteosarcoma metastasis by activating PAX3-MET pathway. Mol Carcinog 2017; 56: 1312-1321. https://doi. org $/ 10.1002 / \mathrm{mc} .22593$

[16] SCHULTE TW, TORETSKY JA, RESS E, HELMAN L, NECKERS LM. Expression of PAX3 in Ewing's sarcoma family of tumors. Biochem Mol Med 1997; 60: 121-126. https://doi.org/10.1006/bmme.1997.2567

[17] OKCU MF, WANG RY, BUESO-RAMOS C, SCHOBER W, WEIDNER D et al. Flow cytometry and fluorescence in situ hybridization to detect residual neuroblastoma cells in bone marrow. Pediatr Blood Cancer 2005; 45: 787-795. https:// doi.org/10.1002/pbc.20428

[18] MARSHALL AD, GROSVELD GC. Alveolar rhabdomyosarcoma - The molecular drivers of PAX3/7-FOXO1-induced tumorigenesis. Skelet Muscle 2012; 2: 25. https://doi. org/10.1186/2044-5040-2-25

[19] MARSHALL AD, VAN DER ENT MA, GROSVELD GC. PAX3-FOXO1 and FGFR4 in alveolar rhabdomyosarcoma. Mol Carcinog 2012; 51: 807-815. https://doi.org/10.1002/ mc. 20848

[20] LI HG, WANG Q, LI HM, KUMAR S, PARKER C et al. PAX3 and PAX3-FKHR promote rhabdomyosarcoma cell survival through downregulation of PTEN. Cancer Lett 2007; 253: 215-223. https://doi.org/10.1016/j.canlet.2007.01.020

[21] GRYDER BE, YOHE ME, CHOU HC, ZHANG X, MARQUES J et al. PAX3-FOXO1 establishes myogenic super enhancers and confers BET bromodomain vulnerability. Cancer Discov 2017; 7: 884-899. https://doi. org/10.1158/2159-8290.CD-16-1297

[22] YIN JQ, GAO J, SHAO R, TIAN WN, WANG J et al. siRNA agents inhibit oncogene expression and attenuate human tumor cell growth. J Exp Ther Oncol 2003; 3: 194-204. https:// doi.org/10.1046/j.1359-4117.2003.01092.x

[23] ZHANG L, XIA L, ZHAO L, CHEN Z, SHANG X et al. Activation of PAX3-MET pathways due to miR-206 loss promotes gastric cancer metastasis. Carcinogenesis 2015; 36: 390-399. https://doi.org/10.1093/carcin/bgv009 